\title{
Composite Materials Containing Perfluorinated and Siloxane Units for Vertical Alignment of Liquid Crystals”
}

\author{
Rasha Ata Alla ${ }^{1}$, Gurumurthy Hegde ${ }^{1,2}$, Lachezar Komitov ${ }^{1 \#}$, Andrea Morelli ${ }^{3}$, Emo Chiellini ${ }^{3}$, \\ Giancarlo Galli ${ }^{3}$
}

${ }^{1}$ Liquid Crystal Group, Department of Physics, University of Gothenburg, Gothenburg, Sweden; ${ }^{2}$ Faculty of Industrial Sciences and Technology, University Malaysia Pahang, Kuantan, Malaysia; ${ }^{3}$ Department of Chemistry and Industrial Chemistry, University of Pisa, Pisa, Italy.

Email: "komitov@physics.gu.se, murthyhegde@gmail.com

Received November $16^{\text {th }}, 2012$; revised December $20^{\text {th }}, 2012$; accepted December $31^{\text {st }}, 2012$

\begin{abstract}
Here we report on the study of vertical alignment of a nematic liquid crystal due to the segregation between perfluorinated and the polysiloxane groups of a multi-component composite alignment material. The measured surface anchoring energy was found to depend on the relative amount of polysiloxane matrix in the composite material. These kind of self-assembly will reduce the preparation time for display by eliminating few steps.
\end{abstract}

Keywords: VA; Nematic; Self Assembling; Vertical Alignment; Anchoring Energy

\section{Introduction}

Liquid Crystal alignment is the subject of interest ever since it is used in the display industry. Many materials capable to align liquid crystals (LC) have been discovered lately and used for preparation of alignment layers in liquid crystal displays (LCDs). Majority of these materials are devoted to vertical alignment (VA) since this kind of alignment offers some advantages such as high contrast ratio and no need for mechanical rubbing. The most important physical mechanism behind VA is the low surface energy of the alignment surface [1]. However, depending on the alignment material structure, the LC molecules may interact sterically with the molecules of the alignment material. As shown in ref. [2], the steric interactions are playing an important role for the quality of VA and the magnitude of the anchoring strength. Considerable attention has been consecrated to self-assembled materials, among all other materials which promote VA, because of their wide applications in advanced materials such as optoelectronic materials since they have the ability for self-assembling into regular manner with Nano sized structures [3]. Perfluorinated groups are widely used for many applications as self-assembled materials since they show very interesting properties such as low surface energy and high chemical and thermal stability [3-7]. It has been observed that the align-

\footnotetext{
* GH is thankful to the Universiti Malaysia Pahant for financial support in the form of RDU grant RDU120367.

${ }^{\#}$ Corresponding author.
}

ment layers with low surface energy, such as the fluorine-rich films, usually gives rise to VA since the surface energy of the alignment layer is less than the surface energy of the liquid crystal $[8,9]$. In general fluorination process enhances the rigidity of the molecules and increases the stability by reinforcing the microphase segregation with the polar groups due to the high electronegativity of the fluorine atoms $[3,4,10]$. In this work perfluorinated groups, regarding their properties, were used as a part of mixture to perform an alignment material as a feasible method to achieve a uniform good VA to fulfill LCDs development requirements.

\section{Experimental}

Here we report on the study of VA of a nematic liquid crystal promoted by multi-component composite alignment materials. For our experimental study, conventional sandwich cells were prepared. The cells' substrates were pre-coated with transparent conductive layer ITO with alignment layer deposited on top of it.

A photopolymerized thin film, prepared from a multicomponent composite material with perfluorinated and polysiloxane groups as major components, was used as alignment layer. The chemical structures of the components of the composite alignment materials, which were studied in this work, are shown in Figure 1.

The relative ratio of these components in the composite material blends are given in Table $\mathbf{1}$.

Perfluorinated siloxane polymers have attracted our 


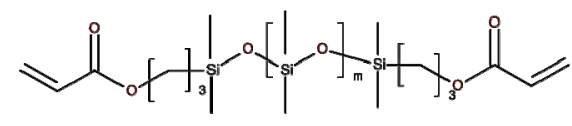

(a)

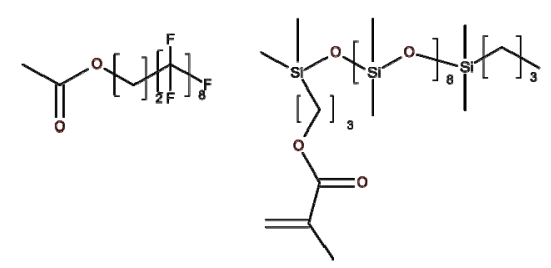

(c)

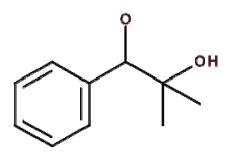

(d)

Figure 1. Compounds used of the composite alignment materials: polysiloxane crosslinker (a), perfluorinated (b) and polysiloxane units (c) and photointiator (d).

Table 1. Alignment materials composition.

\begin{tabular}{ccccc}
\hline \multirow{2}{*}{$\begin{array}{c}\text { Composite } \\
\text { alignment } \\
\text { material }\end{array}$} & \multicolumn{4}{c}{ Components } \\
\cline { 2 - 5 } & $\mathrm{a} \mathrm{wt} \%$ & $\mathrm{~b} \mathrm{wt} \%$ & $\mathrm{c} \mathrm{wt} \%$ & $\mathrm{~d} \mathrm{wt} \%$ \\
\hline Mixture 1 & 83 & 15 & - & 2 \\
Mixture 2 & 54 & 6 & 39 & 1 \\
Mixture 3 & 53 & 16 & 29 & 2 \\
Mixture 4 & - & 89 & 8 & 3 \\
\hline
\end{tabular}

attention since their side perfluorinated groups have the ability to segregate and self-assembled in nano-sized domains when mixed with polysiloxane. In these domains the perfluorinated groups are assembled in a regular fashion, as illustrated in Figure 2, oriented preferably perpendicular to the alignment layer surface.

\section{Sample Preparation}

The composite material was dissolved in polyglycol methyl ether acetate (PGMEA) and 1\% wt solution of the material was spin coated on top of the ITO layer at speed $3000 \mathrm{rpm}$ for 20 seconds. The coated substrates then were baked at $100^{\circ} \mathrm{C}$ for $15 \mathrm{~min}$ to evaporate the solvent. UV photopolymerization was carried out at room temperature by normal incident of a non-polarized UV light at an energy level of about $20 \mathrm{~mJ} / \mathrm{cm}^{2}$ for different exposure times. The prepared experimental cells were of conventional sandwiched type with cell gap of about 3 $4 \mu \mathrm{m}$.

Nematic liquid crystal MLC $6608(\Delta \varepsilon<0)$ (Merck) was injected into the cells. A reference cell with alignment layer made from the VA material SE-1211 (Nissan) dissolved in N-Methyl-2-Pyrrolidinone (NMP) with concentration of $5 \% \mathrm{wt}$, was prepared too.

\section{Results and Discussion}

Very good and uniform VA was found in the cells at ex-

posure time of $20 \mathrm{~min}$. Figure 3 shows the cartoon diagram of the orientation of the molecules promoted by the multi-component composite alignment materials.

The steric interactions between the self-assembled fluorinated groups and the liquid crystal molecules results in stronger and more temperature stable vertical alignment. The alignment quality and the electro-optic characteristics of the cells were studied in a Zeiss microscope between crossed polarizers using a standard electro-optical set-up.

The measured values of the response times $\tau_{\text {off }}$ and $\tau_{\text {on }}$ are presented in Table 2. The polar anchoring energy $W$ was calculated using the Equation [11]:

$$
\tau_{\text {off }} \approx 4 \gamma_{1} d / W \pi^{2}
$$

where, $\gamma_{1}$ is the rotational viscosity, $d$ is the cell gap.

The polar anchoring energy measured in the cells with alignment layer made from the composite materials seems to be as strong as the one measured in the reference cell (c.f. Table 2).

Due to the segregation of the compounds, nano-sized domains containing perfluorinated groups are formed in the composite alignment material. The study of such composite materials by NEXAFS shows that the fluorinated groups are self-assembled in the way shown in Figure 2 making an angle of about $45^{\circ}$ between each

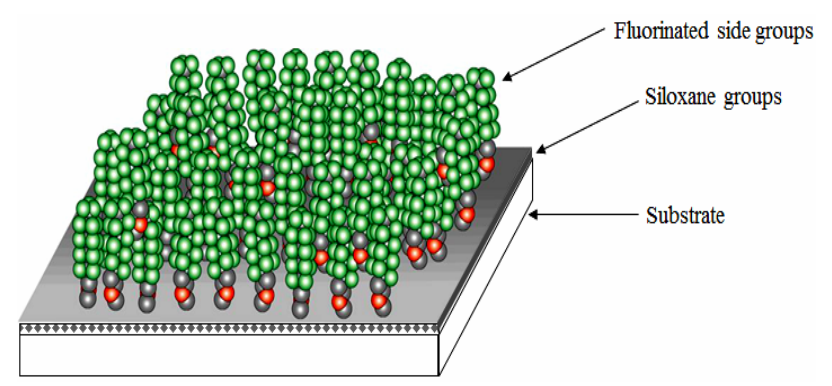

Figure 2. Schematic presentation of the phase separation and the self-organisation in the multi-component composite alignment materials. Model built up on the information gained by NEXAFS.

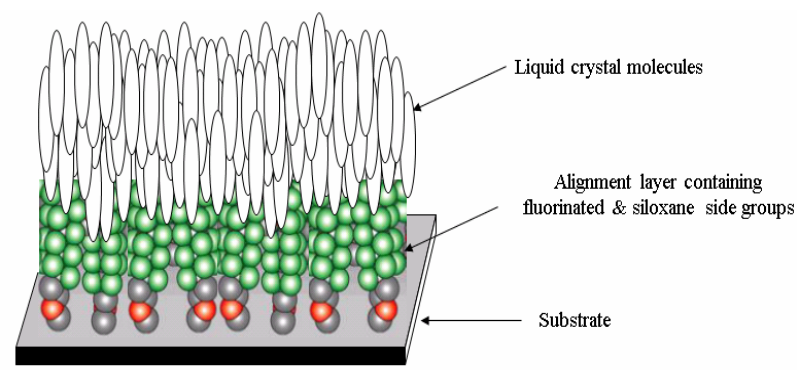

Figure 3. Schematic presentation of the liquid crystal alignment promoted by the multi-component composite alignment material. The steric interactions between the self-assembled fluorinated groups and the liquid crystal molecules results in stronger and more temperature stable VA. 
Table 2. Fall $\tau_{\text {fall }}$ and rise $\tau_{\text {rise }}$ time, and polar anchoring energy $W$.

\begin{tabular}{ccccc}
\hline $\begin{array}{c}\text { Composite } \\
\text { alignment } \\
\text { material }\end{array}$ & $\boldsymbol{\tau}_{\text {off }} \mathbf{m s}$ & $\boldsymbol{\tau}_{\text {on }} \mathbf{m s}$ & $\boldsymbol{d} / \boldsymbol{\tau}_{\text {off }} \times \mathbf{1 0}^{-\mathbf{3}} \mathbf{~ \mathbf { s } / \mathbf { s }}$ & $\begin{array}{c}\text { Anchoring } \\
\mathbf{e n e r g y} \\
\mathbf{J} / \mathbf{m}^{2} \times \mathbf{1 0}^{-5}\end{array}$ \\
\hline Mixture 1 & 10 & 38 & 0.320 & 2.4 \\
Mixture 2 & 14 & 24 & 0.264 & 2 \\
Mixture 3 & 12 & 32 & 0.283 & 2.2 \\
Mixture 4 & 22 & 60 & 0.205 & 1.5 \\
Ref. material & 8.82 & 9.36 & 0.453 & 2.17 \\
SE1211 & & & & \\
\hline
\end{tabular}

other [4]. Thus, the steric interactions between the selfassembled fluorinated groups, oriented perpendicular to the alignment layer surface, promote uniform, stronger and more temperature stable vertical alignment. Figure 3 shows the way molecules oriented by the multi-component composite alignment materials. Hence, two major interactions are taking part in the VA of the LC promoted by the composite alignment layer; one-due to the low surface energy of the polysiloxane as well as of the fluorinated groups, and the second one due to the steric interactions between the self-assembled fluorinated groups and the LC molecules.

It is also found that the relative concentration of the polysiloxane and perfluorinated compounds in the composite alignment materials is of importance for the alignment characteristics of the layers made from these materials enabling thus an efficient control of the properties of the alignment layers.

\section{Conclusion}

A promising new series of photopolymerizable composite materials appropriate for promoting VA in LCDs with very good quality, suitable thermal stability and anchoring strength, is presented. The curing time for these materials can be adjusted and reduced by controlling the relative amounts of the components used in the composite mixtures. The high quality VA, promoted by most of these composite alignment materials and the strong polar anchoring, seems to be a result of the peculiar nanostructured surface.

\section{REFERENCES}

[1] J. Cognard, "Alignment of Nematic Liquid Crystals and Their Mixtures," Molecular Crystals and Liquid Crystals,
Supplement Series, Vol. 1, 1982, pp. 1-78.

[2] V. Fazio, F. Nanneli and L. Komitov, "Sensitive Methods for Estimating the Anchoring Strength of Nematic Liquid Crystals on Langmuir-Blodgett Monolayers of Fatty Acids," Physical Review E, Vol. 63, 2001, pp. 1-8, Article ID: 061712 .

[3] D. K. Yoon, S.-H. Park, E. H. Lee, Y. H. Kim, P. Stenger, J. A. Zasadzinski and H.-T. Jung, "Surface Ordering of a Perfluorinated Self-Assembled, Dendrimer on a Water Subphase," Langmuir, Vol. 21, No. 11, 2005, pp. 4989 4995.

[4] X. F. Li, L. Andruzzi, E. Chiellini, G. Galli, C. K. Ober, A. Hexemer, E. J. Kramer and D. A. Fischer, "Semifluorinated Aromatic Side-Group Polystyrene-Based Block Copolymers: Bulk Structure and Surface Orientation Studies," Macromolecules, Vol. 35, No. 21, 2002, pp. 8078-8087. doi:10.1021/ma020463k

[5] D. R. Iyengar, S. M. Perutz, C.-A. Dai, C. K. Ober and E. J. Kramer, "Surface Segregation Studies of FluorineContaining Diblock Copolymers," Macromolecules, Vol. 29, No. 4, 1996, pp. 1229-1234.

[6] Y. Katano, H. Tomono and T. Nakajima, "Surface Property of Polymer Films with Fluoroalkyl Chains," Macromolecules, Vol. 27, No. 8, 1994, pp. 2342-2344. doi: $10.1021 / \mathrm{ma} 00086 \mathrm{a} 060$

[7] J. Hopken and M. Mdller, "Low-Surface-Energy Polystyrene," Macromolecules, Vol. 25, No. 5, 1992, pp. 1461-1467. doi:10.1021/ma00031a016

[8] H. J. Ahn, S. J. Rho, K. C. Kim, J. B. Kim, B. Hwang, C. J. Park and H. K. Baik, "Ion-Beam Induce Liquid Crystal Alignment on Diamond-Like Carbon and Fluorinated Diamond-Like Carbon Thin Films," Japanese Journal of Applied Physics, Vol. 44, No. 6A, 2005, pp. 4092-4097. doi:10.1143/JJAP.44.4092

[9] H. J. Ahn, J. B. Kim, K. C. Kim, B. H. Hwang, J. T. Kim, H. K. Baik, J. S. Park and D. Kang, "Liquid Crystal Pretilt Angle Control Using Adjustable Wetting Properties of Alignment Layers," Applied Physical Letters, Vol. 90, No. 25, 2007, pp. 253505-253507. doi:10.1063/1.2749843

[10] T. Imae, T. Takeshita and M. Kato, "Phase Separation in Hybrid Langmuir-Blodgett Films of Perfluorinated and Hydrogenated Amphiphiles. Examination by Atomic Force Microscopy," Langmuir, Vol. 16, No. 2, 2000, pp. 612-621. doi:10.1021/1a9902237

[11] X. Nie, R. Lu, H. Xianyu, T. X. Wu and S.-T. Wu, “Anchoring Energy and Cell Gap Effects on Liquid Crystal Response Time," Journal of Applied Physics, Vol. 101, 2007 , pp. $1-5$. 